\title{
Image based fog detection in vehicles
}

\author{
Mario Pavlić, Heidrun Belzner, Gerhard Rigoll and Slobodan Ilić
}

\begin{abstract}
Modern vehicles are equipped with many cameras and their use in many practical applications is extensive. Detecting the presence of fog from images of a camera mounted in vehicles is a very challenging task with the potential to be used in many practical applications. Approaches introduced until now analyze properties of local objects in the image like lane markings, traffic signs, back lights of vehicles in front or head lights of approaching vehicles. By contrast to all these related works we propose to use image descriptors and a classification procedure in order to distinguish images with fog present from those free of fog. These image descriptors are global and describe the entire image using Gabor filters at different frequencies, scales and orientations. Our experiments demonstrated hight potential of the proposed method for fog detection on daytime images.
\end{abstract}

\section{INTRODUCTION}

Camera based driving assistance systems are one of the core technology trends in intelligent vehicles. Well known applications are, for example, the lane departure warning, the road sign detection or the high beam assistance. All these systems detect tangible objects in the camera images like lane markings, road signs, light sources of the vehicles ahead or forthcoming vehicles. Detecting context information describing the environment surrounding, like fog, rain or snow, is still very challenging. In this paper we address the problem of fog detection. Previous camera based fog detection systems analyze therefore distinct objects in the image [7] or image regions like the road region [4], [7], [18] or the horizon [3], [11], i.e. the position where the sky touches the road. However, these approaches are not reliable for everyday use, because the road or the horizon is often occluded by other objects like vehicles or bridges, while the features like lane markings can be of different quality, e.g freshly painted markings significantly differ from old ones. The only stable information that we can always see in images of foggy weather conditions is a decrease in contrast and blurring in the whole image. For that reason we propose a new method to detect such an event by using global image features. Thereby we analyze the power spectrum being the squared magnitude of the Fourier transform of the image that holds information about the frequencies in the image discarding spatial information. From the power spectrum we

M. Pavlić is with BMW Group, Traffic Technology and Traffic Management, 80788 Munich, Germany Mario.Pavlic@bmw. de

H. Belzner is with BMW Group, Traffic Technology and Traffic Management, 80788 Munich, Germany Heidrun. Bel zner@bmw. de

G. Rigoll is with Department of Human-Machine-Communication, Technische Universität München, Theresienstr. 90, 80333 Munich, Germany Rigolletum.de

S. Ilić is with Department of Informatics, Technische Universität München, Boltzmannstr. 3, 85748 Garching, Germany Slobodan.Ilicein.tum.de build the image features that can be then fed to the classifier trained on fog and fog free images. As a result we obtained high recognition rates on about $1 \mathrm{~h}$ of test videos.

There are many applications which could benefit from this information, some of these are:

- Advice on implicit speed limit. Speed limits are either given explicit by signs or implicit by traffic regulations. For example if the visibility range is less then $50 \mathrm{~m}$, the speed is restricted to $50 \mathrm{~km} / \mathrm{h}$. If the vehicle would be able to recognize such a situation, the driver could be notified to this regulation.

- Fog lamp/rear fog lamp assistant. If the presence and occurrence of fog could be recognized by vehicles the fog and rear fog lamp could be automatically switched on and off, as it is often done for low beam light by nowadays vehicles.

- Strategy adjustment of the high beam assistance. The high beam assistance supports the driver by switching on and off the high beam. In the presence of fog the driver gets dazzled by his own high beam. If the vehicle could detect fog at night the high beam assistant could be adjusted accordingly.

- Optimizing image based detection algorithms. Fog causes a decrease in contrast and blurring in images. If such images could be detected beforehand image restoration algorithms could perform a preprocessing to optimize object based recognition processes.

- Local Hazard Warning. The Local Hazard Warning tries to countervail hazardous situations by using among others information from vehicles being currently on the road and precisely warns drivers heading toward such locations. A vehicle being in a situation with dense fog should detect it and send this information to a central back-end that can be distributed to other road users.

In the following we present our new approach for image based fog detection (chapter IV) and the results of our evaluation (chapter V). Prior to that we will regard the terms fog and visibility range (chapter III) as well as previous works (chapter II). At the end we will give a conclusions and look on future works (chapter VI).

\section{Previous Works}

In [4] C. Busch and E. Debers introduced an approach to determine the visibility range under foggy weather conditions from images of stationary traffic management systems. After a manual masking of the road they performed a wavelet based contrast measurement. The image line, upon which no contrast higher than $5 \%$ appears, defines the visibility range in the camera image. From the known camera configuration 
relative to the road a transformation from image to world coordinates was done. In the range from 300 to $1000 \mathrm{~m}$ the proposed system outputs a visibility range in $50 \mathrm{~m}$ steps, in the range of less than $300 \mathrm{~m}$ in $10 \mathrm{~m}$ steps. Even if this system was designed for a stationary use it is interesting in our field of application, as with existing image processing techniques, like an appropriate lane and object detection, it could be applicable for the use in vehicles.

In [18] D. Pomerleau introduced a system to estimate the visibility range through the use of a camera mounted inside a vehicle. His system is generic and aims to cover all possible situations of low visibility caused by dazzling, rain, snow or fog. The visibility range is thereby estimated by the attenuation of the contrast along similar road features like lane markings, banquet or even oil stripes. Initially a trapezoid region containing the road ahead is extracted and warped to the bird-eye-view. Then by shifting the rows of the image on this warped view a straight road profile is obtained. The horizontal intensity profile of this image is analyzed at different image rows and from the difference in intensity peaks of the upper and lower rows the contrast attenuation is estimated. This contrast change usually happens around road features like lane markings.

In [11] Hautière et al. proposed a method for fog detection form images captured by a camera mounted inside vehicles. It is based on Duntley's law for the contrast attenuation $C=C_{0} e^{-k d}$ [15]. Here, $C$ is the perceived contrast at the distance $d$ from an object with the intrinsic contrast of $C_{0}$ and $k$ is the extinction coefficient that characterizes the visibility conditions. Under the assumption that a contrast less than $5 \%$ could not be perceived $(C=0.05)$ and that we analyze a black object against a white background $\left(C_{0}=1\right)$, Duntley's law could be rewritten as the visibility range as a function of the extinction coefficient $d_{v}=-\frac{1}{k} \ln (0.05)$. The proposed method now determines the extinction coefficient based on the inclination point of an intensity function, which models the intensity flow from the road to the sky. This method is based on a modified region growing approach used to find a region containing parts of the road and the sky. Inside this region a measurement range is defined and the median intensity value for each image row is determined. Based on the inclination point of the so obtained intensity function the extinction coefficient is calculated and hence the visibility range estimated. By perspective projection and assuming a flat world the system outputs a metric estimation of the visibility range. If the estimation of visibility range delivers a value of infinity, the authors deduce that there is no fog. Because it is not always guaranteed to find a region containing parts of the road and the sky, the authors proposed a modified version of their algorithm based on the approach of Pomerleau [18] where information of lane markings are incorporated [13]. Furthermore they introduced an approach based on stereo camera systems to avoid the limitations and inaccuracies from the mono camera approach and the flat world assumption [12].

Another approach to estimate the visibility range under foggy weather conditions was proposed by Bronte et al. [3].
They extract two adjacent image regions, a road and a sky region, each with a region growing algorithm. Furthermore they determine the current vanishing point respectively the vanishing line from image features. If the vanishing point is inside the upper sky region, they deduce the presence of fog. Then the image row, where the two regions touch, provides the current visibility range. By perspective projection and assuming a flat world they calculate the visibility range in meter. To avoid false detections they use a kind of threshold based preceded no fog detector which sums the magnitudes of gradients in the upper half image region. Because the estimation of the visibility range based on a single image is very unstable, i.e. it exhibits large variations in time, they calculate the median visibility range over a specific period of time.

While all methods presented until now are based on daytime scenes, Gallen et al. introduced in [7] an aproach for nighttime scenes. They divide the problem in two categories, scenes containing light sources like streetlights, ahead or forthcoming vehicles and scenes without external light sources. In the first category they investigate the light propagation around light sources. If the corona of a light source has a sharp transition to the surrounding they conclude that there is no fog, otherwise that there is fog. The translation is thereby examined by a multistage threshold based approach. Having a scene without external light sources they analyze the propagation of light of the vehicle headlights. For that purpose they recorded templates for their experimental vehicle at different visibility condition in a fog chamber. So each template also includes a specific visibility range value. Their method now averages a specific number of images and compares this average image with all templates. The template with the smallest euclidean distance in pixel values finally provides the visibility range.

In contrast to above mentioned approaches that rely on localized image features related to lane markings, road profiles etc. we introduce a classification scheme based on global image features where the classifier is trained on fog and fog-free images.

\section{Remarks on the Definition of Fog AND VISIBILITY RANGE}

According to the international definition in meteorology fog is defined as a cloud touching the ground. If the result is a visibility reduction of less than $1000 \mathrm{~m}$ it is called fog, otherwise mist [1]. The visibility range is thereby defined as the longest distance, at witch a black object of adequate size could be seen against the sky. For our application this definition of fog is unrewarding, because a driver is not affected by fog until the visibility range decreases considerably more. Hence a fog detection system for driver assistance should also react on visibility ranges less than $1000 \mathrm{~m}$. To derive a definition for fog in our application, we refer to the German road traffic regulations (StVO). According to the StVO a road user is only allowed to drive as fast as he is able to control his vehicle. In particular he has to adjust his driving speed on the current road, traffic, visibility and weather conditions. 


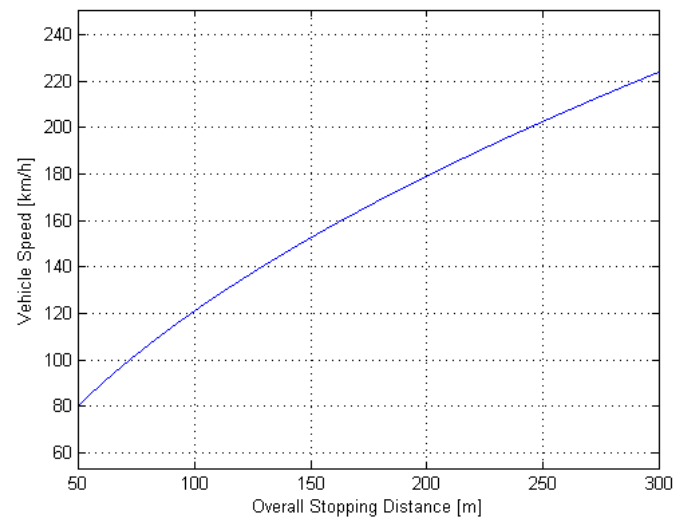

Fig. 1. Illustration of the overall stopping distance subject to the vehicle speed, resp. recommended maximum speed subject to current visibility range.

Assuming a driver controls his vehicle when he is able to bring it to a halt within the current visibility range, we could derive a definition for fog by the relation between the current vehicle speed and the resulting overall stopping distance. The overall stopping distance $d=d_{R}+d_{S}$ is defined as the sum of the distance covering while reaction time $d_{R}=v \cdot t_{R}$ and the stopping distance $d_{S}=\frac{v^{2}}{2 a}$. Thereby $v$ describes the vehicle speed, $t_{R}$ the reaction time and $a$ the deceleration. Under the simplified assumption of a constant deceleration during the braking action the vehicle speed could be written as a function of the overall stopping distance

$$
v(d)=\frac{-t_{R}+\sqrt{t_{R}^{2}+2 d / a^{2}}}{1 / a^{2}} .
$$

Assuming $t_{R}=0.8 \mathrm{~s}$ as mean reaction time and $a=$ $7.716 \mathrm{~m} / \mathrm{s}^{2}$ as mean deceleration ${ }^{1}$ we get the current speed as a function of the overall breaking distance as shown in fig. 1. Thereby the overall breaking distance is plotted along the ordinate and the vehicle speed along the abscissa.

That is how it becomes obvious that e.g. a vehicle speed of $200 \mathrm{~km} / \mathrm{h}$ leads to an overall breaking distance of a bit less than $250 \mathrm{~m}$. On the other side, if substituting the overall braking distance by the visibility range, we can see from this figure, that at a visibility range of e.g. $100 \mathrm{~m}$ one should not drive faster than $120 \mathrm{~km} / \mathrm{h}$. So we derive following fog categories:

- Visibility distance above $1000 \mathrm{~m}$ : No Fog

- Visibility distance between 300 and 1000 m: Low Fog

- Visibility distance between 100 and 300 m: Fog

- Visibility distance below $100 \mathrm{~m}$ : Dense Fog

Depending on the specific application different fog categories are relevant. E.g. the fog categories Fog and Dense Fog are relevant for the high beam assistant whereas for the Local Hazard Warning it's only Dense Fog.

\footnotetext{
${ }^{1}$ The values for the mean reaction time and the mean deceleration originate from an example of the Bavarian police, taken from their website http://www.polizei.bayern.de/verkehr/studien/index.html/31494 on 28th October 2011.
}

\section{Spectral Fog Detection}

Studies in scene perception have shown that observers are able to recognize real-world scenes at a single glance [19], [21]. In an Rapid Serial Visual Presentation (e.g. RSVP with $100 \mathrm{~ms}$ exposure per image) they were able to detect an image, just given a description like "birthday party". The phenomena of understanding everything at once, regardless of the visual complexity of the scene, refers to the gist of a scene [20]. In image processing different approaches were introduced to perform simple classification tasks based on the gist of the scene by using global image features [8], [9], [16], [22], [24], [25]. Furthermore Oliva and Torralba presented a possibility to classify natural images in semantic classes [17], [23]. It turns out, that the energy spectrum of an image, the squared magnitude of the Fourier transform, is a good choice for global features, because it doesn't contain any spatial information. Fig. 2 shows different scenes under foggy and clear weather conditions as well as the corresponding power spectra. In the case of a fog scene the frequency components are concentrated at the zero frequency whereas in a fog free scene one finds a broadly spread spectrum. The reason for that is contrast attenuation and blurring in the image caused by fog. While sharp edges are modeled by many low and high frequencies, smooth edges consist of only low frequencies. Now it's a matter of working out these differences in spectra by appropriate methods from image processing. We first normalize the input image $i(x, y)$ in a prefiltering step. Afterwards we perform a feature extraction that is based on the calculation of the power spectrum of the image followed by two-stage feature reduction done in terms of a Gabor filter bank sampling and Principal Components Analysis (PCA). Finally we use a Support Vector Machine (SVM) to perform the classification task. Fig. 3 summarizes our single steps.

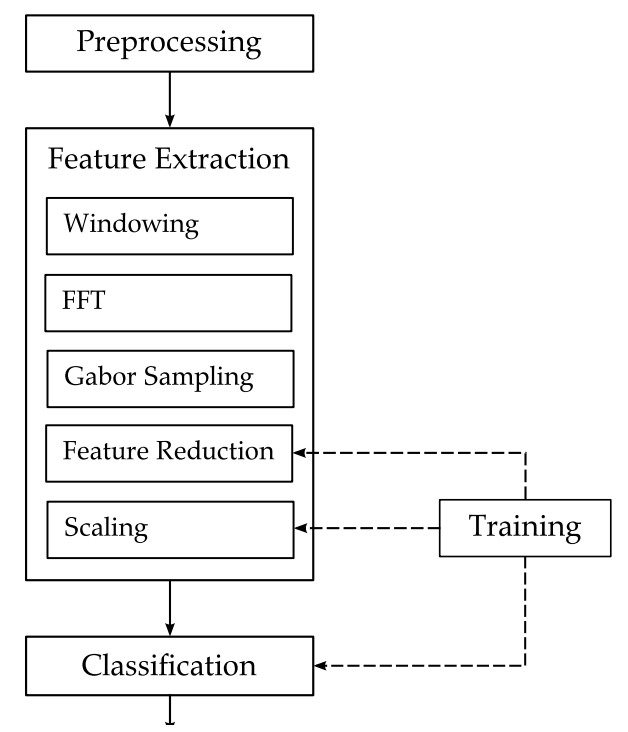

Fig. 3. Summery of the single steps for fog detection. 

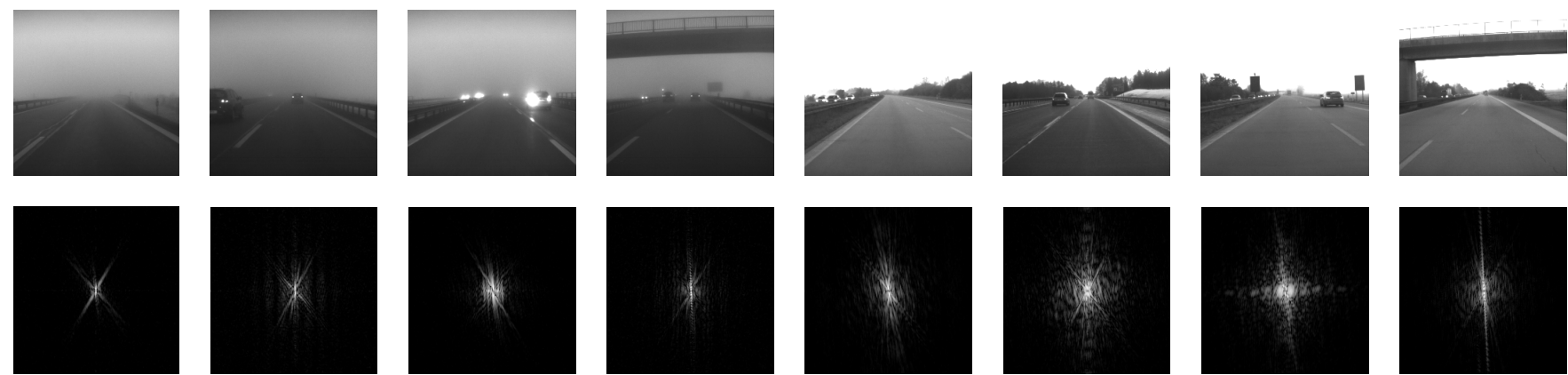

Fig. 2. Images of fog and fog free scenes and the corresponding power spectra. The power spectra are displayed in logarithmic unit, with zero frequency in the middle and higher frequencies at the border. The images presented above show clear differences in the spectra between similar scenes. While in fog scenes the frequency components are concentrated at the origin, in fog free scenes they are broadly spread.

\section{A. Preprocessing}

To avoid that some image regions dominate the spectrum and to reduce illumination effects we first perform a normalization:

$$
i^{\prime}(x, y)=\frac{i(x, y) * h(x, y)}{\varepsilon+\sqrt{[i(x, y) * h(x, y)]^{2} * g(x, y)}} .
$$

Thereby $g(x, y)$ describes an isotropic low pass filter with a radial cut off frequency at 0.015 lines/pixel and $h(x, y)=$ $1-g(x, y)$. The numerator is a high pass filter that cancels the mean intensity of the image. The denominator acts as a local estimator of the variance of the output of the high pass filter. $\varepsilon$ is a constant that avoids noise enhancements in homogeneous image regions, we therefore used the experimental determined value $\varepsilon=10$. For the normalization we use a square image section and equalize the intensity values to the range $[0,255]$.

\section{B. Feature Extraction}

For the classification we model the variation of fog and fog free scenes in the power spectrum

$$
\Gamma\left(f_{x}, f_{y}\right)=\left|I\left(f_{x}, f_{y}\right)\right|^{2},
$$

which is defined by the square magnitude of the Fourier transform for every frequency $f_{x}$ and $f_{y}$ of the image given by

$$
I\left(f_{x}, f_{y}\right)=\sum_{x=0}^{N-1} \sum_{y=0}^{N-1} i^{\prime}(x, y) e^{-j 2 \pi\left(f_{x} x+f_{y} y\right)}
$$

of the prefiltered, square image section of size $N$.

Before the Fourier transform we perform a windowing with a two dimensional Hanning window to avoid broadband signal components along the axis, which would result from the implicit assumption of a periodically continued signal [10]. Because of the symmetry in the power spectrum we hence get total of $N^{2} / 2$ features. With regard to an accurate classification we perform a two stage feature reduction by sampling the spectrum and a subsequently Principal Components Analysis.

The Gabor sampling is done by a filter bank of scaled and oriented filters. The $i$ th Gabor filter is defined as

$$
G_{i}\left(f_{x}, f_{y}\right)=e^{-2 \pi^{2}\left(\sigma_{x}^{2}\left(f_{x}^{\prime}-f_{r}\right)^{2}+\sigma_{y}^{2} f_{y}^{\prime 2}\right)}
$$

with $f_{x}^{\prime}=f_{x} \cos (\Theta)+f_{y} \sin (\Theta)$ and $f_{y}^{\prime}=-f_{x} \sin (\Theta)+$ $f_{y} \cos (\Theta)$. Thereby the filters are shifted to the position $f_{r}$ and rotated by $\Theta$ so that they are positioned semicircular around the zero frequency at different radii. We used a filter bank with 10 frequency bands and a decreasing number of orientations, from 24 in the outer to 4 in the inner band. Other configurations, e.g. 5 frequency bands, each with 12 orientations, yield to similar results (see fig. 4).

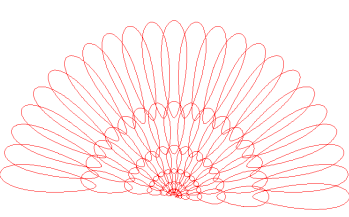

(a)

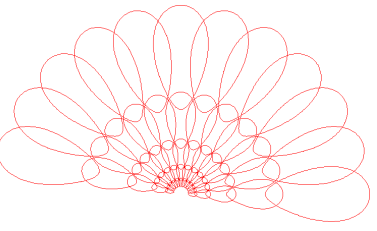

(b)
Fig. 4. Example for two configurations of Gabor filter banks. (a) 100 Gabor filters with 10 scales, each with $24,16,12,12,8,8,6,6,4$ resp. 4 orientations. (b) 60 Gabor filters with 5 scales, each with 12 orientations.

The Gabor features are used for the subsequent PCA. Therefore we determine the Gabor features

$$
g_{i}=\sum \sum \Gamma\left(f_{x}, f_{y}\right) G_{i}\left(f_{x}, f_{y}\right), i=1,2 \ldots K
$$

from $M$ images, i.e. from our training data consisting of an equivalent number of fog and fog free images, and collect them in the Gabor feature vector $\mathbf{g}=\left\{g_{i}\right\}_{i=1}^{K}$. The size of this feature vector is equal to the number of Gabor filters $K$. Afterwards we determine the mean Gabor feature vector

$$
\overline{\mathbf{g}}=\frac{1}{M} \sum_{m=1}^{M} \mathbf{g}_{m}
$$

and the covariance matrix

$$
\mathbf{C}=\frac{1}{M} \sum_{m=1}^{M} \boldsymbol{\phi}_{m} \boldsymbol{\phi}_{m}^{T}=\frac{1}{M} \mathbf{A} \mathbf{A}^{T}
$$

with $\phi_{m}=\mathbf{g}_{m}-\overline{\mathbf{g}}$ and $\mathbf{A}=\left[\phi_{1} \phi_{2} \ldots \phi_{M}\right]$ and perform the eigenvalue decomposition

$$
\mathbf{V}^{-1} \mathbf{C V}=\mathbf{D} .
$$

Thereby the diagonal matrix $\mathbf{D}$ contains the eigenvalues and $\mathbf{V}=\left[\begin{array}{llll}\mathbf{v}_{1} & \mathbf{v}_{2} & \ldots & \mathbf{v}_{K}\end{array}\right]$ the corresponding eigenvectors, the so called principal components. The eigenvalues are sorted in 
descending order. Now, for the feature reduction, we use the first $N, N<K$ eigenvectors and collect them in the matrix $\mathbf{W}=\left[\begin{array}{llll}\mathbf{w}_{1} & \mathbf{w}_{2} \ldots \mathbf{w}_{N}\end{array}\right]$. We therefore used the first $N=6$ eigenvectors. Now the reduced feature vector is given by

$$
\widetilde{\boldsymbol{\omega}}=\mathbf{W}^{T}(\mathbf{g}-\overline{\mathbf{g}}) \text {. }
$$

Before using these features for the classification respectively training task we perform a scaling of each feature to the range of $[-1,1]$. The scale coefficients are gathered in the scale matrix $\mathbf{S}=\operatorname{diag}\left\{s_{i}\right\}_{i=1}^{L}$. From feature extraction we finally get the global feature vector

$$
\begin{aligned}
\boldsymbol{\omega} & =\mathbf{S} \widetilde{\boldsymbol{\omega}} \\
& =\mathbf{S}\left(\mathbf{W}^{T}(\mathbf{g}-\overline{\mathbf{g}})\right) .
\end{aligned}
$$

\section{Classification}

For classification we used a SVM with a Radial Basis Function (RBF) kernel

$$
K\left(\boldsymbol{\omega}_{i}, \boldsymbol{\omega}\right)=e^{-\gamma\left|\boldsymbol{\omega}_{i}-\boldsymbol{\omega}\right|^{2}} .
$$

were $\omega_{i}, i=1, \ldots, M$ are the feature vectors from the training set and $\omega$ is the feature vector of the image to be classified. The regularization parameter $C$ and $\gamma$ were determined by the training data.

\section{Evaluation}

For the evaluation we used the front camera of the current BMW 5 series (F10), mounted behind the rear mirror parallel to the vehicles longitudinal axis and showing in the driving direction. The camera system provides images with a resolution of $320 \times 240$ pixels at a rate of $60 \mathrm{fps}^{2}$.

Based on this system we recorded images with daytime fog situations on highways. Afterwards the single images were labeled in the categories ${ }^{3}$ Excluded, No Fog, Low Fog, Fog and Dense Fog, as defined in section III.
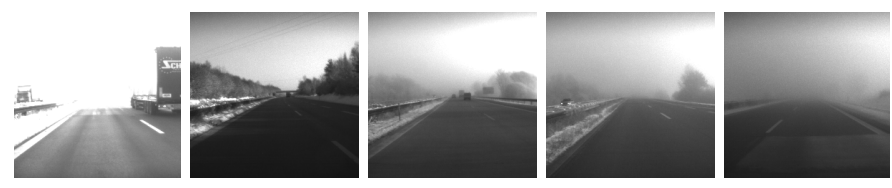

Fig. 5. Example images for labeling categories. Form left to right: Excluded, No Fog, Low Fog, Fog and Dense Fog.

For the labeling we plotted in every image a horizontal line at a distance of $100 \mathrm{~m}$ and $300 \mathrm{~m}$. The pixel coordinates of these lines were calculated by perspective projection from the known camera setup and by assuming a flat world, i.e. that the road is planar. The images are then manually labeled to one of the categories according to the possibility to visually recognize any contrast above predefined distances. In our case we labeled the image as Dense Fog if no contrast could not be observed above the $100 \mathrm{~m}$ distance. This was done for all recorded images.

\footnotetext{
${ }^{2}$ The camera system performs a time multiplexing for different image processing functions. A single function, like the lane detection, works at 15 fps.

${ }^{3}$ The categoriy Excluded contains images with overexposure or dazzle.
}

To determine the overall accuracy, the true positive ${ }^{4}$ and the true negative ${ }^{5}$ rate of our method we used a 8-fold crossvalidation. The recorded data was split into eight equal sized sample sets with the same number of fog and fog free images. Each sample set consists of 5,500 images, composed of 2,750 fog and 2,750 fog free images. As fog images we used Dense Fog labeled images, as fog free images 500 Low Fog and 2, 250 No Fog labeled images. By the composition of the sample sets we payed attention to group similar highway types. E.g. the German highway A8 is, in contrast to the A92, hilly and has a distinct vegetation, which yields to a higher image contrast at clear weather conditions. This means we wanted also to see how the system behaves if we train it e.g. with images of a highway type with basically higher contrasts like the A8 and test it with images of a highway type with low contrasts like the A92. Finally the classifier was trained with one sample set and tested with each of the remaining seven. This was repeated until each sample set was used ones as training data. The overall accuracy, true positive and true negative rate was determined by averaging all results, these are $94 \%, 93 \%$ respectively $96 \%$. The single evaluation results are shown in table I.

\section{CONCLUSIONS AND Future Works}

We demonstrated how the presence of dense fog in images of a daytime driving scene can be recognized by a vision system. Instead of analyzing local features like lane markings or the road region, we used global features in terms of the power spectrum of the Fourier transform. The evaluation results on the basis of 44,000 images showed the potential of our method. Fog free images were classified with $96 \%$ as fog free and fog images were callsified in $93 \%$ as fog images. In total we we achieved an overall accuracy of $94 \%$. Here is to be considered, that we only used No Fog, Low Fog and Dense Fog labeled images and that the total data set does not cover all road profiles. This is important for a well parametrized classifier, but, as a future step, we have to analyze the behavior for images from other categories too. Furthermore another interesting question is if and how this method would work on night-time scenes.

\section{REFERENCES}

[1] American Meteorology Glossary. "Glossary of Meteorology", Online available at http://amsglossary.allenpress.com/glossary. Visited on 28.11.2011.

[2] B. E. Boser, I. M. Guyon, and V. N. Vapnik. "A training algorithm for optimal margin classifiers", In D. Haussler, 5th Editor, Annual ACM Workshop on COLT, 1992, pp. 144-152.

[3] S. Bronte, L.M. Bergasa, L., and P.F. Alcantarilla, "Fog Detection System Based on Computer Vision Techniques", IEEE Intelligent Transportation Systems, vol. 12, 2009, pp. 3-7.

[4] C. Busch and E. Debes,"Wavelet Transform for Analyzing Fog Visibility", IEEE Intelligent Systems, vol. 13(6), 1998, pp.66-71.

[5] C. Cortes and V. Vapnik, "Support-vector network", Machine Learning, vol. 20, 1995, pp.273-297.

[6] R.A. Fisher, "The use of multiple measurements in taxonomic problems", Annals Eugen., Vol. 7, 1936, pp.179-188.

\footnotetext{
${ }^{4}$ Images labeled and detected as fog images.

${ }^{5}$ Images labeled and detected as fog free images.
} 


\begin{tabular}{|c|c|c|c|c|c|c|c|c|c|c|}
\hline & \multicolumn{8}{|c|}{ Test Data } & \multirow[b]{2}{*}{$\sum$} \\
\hline & & $\mathcal{S}_{1}$ & $\mathcal{S}_{2}$ & $\mathcal{S}_{3}$ & $\mathcal{S}_{4}$ & $\mathcal{S}_{5}$ & $\mathcal{S}_{6}$ & $\mathcal{S}_{7}$ & $\mathcal{S}_{8}$ & \\
\hline \multirow{24}{*}{ 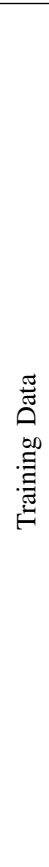 } & \multirow{3}{*}{$\mathcal{S}_{1}$} & & 0.9080 & 0.9667 & 0.9800 & 0.9522 & 0.9585 & 0.9349 & 0.9407 & 0.9487 \\
\hline & & & 0.8564 & 0.9691 & 0.9822 & 0.9622 & 0.9665 & 0.9335 & 0.9150 & 0.9407 \\
\hline & & & 0.9596 & 0.9643 & 0.9778 & 0.9422 & 0.9505 & 0.9364 & 0.9665 & 0.9568 \\
\hline & \multirow{3}{*}{$\mathcal{S}_{2}$} & 0.9695 & & 0.9556 & 0.9651 & 0.9065 & 0.9022 & 0.8765 & 0.8362 & 0.9159 \\
\hline & & 0.9418 & & 0.9480 & 0.9782 & 0.9353 & 0.9156 & 0.8385 & 0.7982 & 0.9079 \\
\hline & & 0.9971 & & 0.9633 & 0.9520 & 0.8778 & 0.8887 & 0.9145 & 0.8742 & 0.9239 \\
\hline & \multirow{3}{*}{$\mathcal{S}_{3}$} & 0.9109 & 0.8771 & & 0.9938 & 0.9700 & 0.9831 & 0.9145 & 0.9369 & 0.9409 \\
\hline & & 0.8233 & 0.7611 & & 0.9902 & 0.9727 & 0.9738 & 0.9502 & 0.9476 & 0.9170 \\
\hline & & 0.9985 & 0.9931 & & 0.9975 & 0.9673 & 0.9924 & 0.8789 & 0.9262 & 0.9648 \\
\hline & \multirow{3}{*}{$\mathcal{S}_{4}$} & 0.9289 & 0.8729 & 0.9887 & & 0.9576 & 0.9898 & 0.9302 & 0.9267 & 0.9421 \\
\hline & & 0.8578 & 0.7571 & 0.9931 & & 0.9851 & 0.9862 & 0.9618 & 0.9651 & 0.9295 \\
\hline & & 1.0000 & 0.9887 & 0.9844 & & 0.9302 & 0.9935 & 0.8985 & 0.8884 & 0.9548 \\
\hline & \multirow{3}{*}{$\mathcal{S}_{5}$} & 0.9333 & 0.8695 & 0.9905 & 0.9965 & & 0.9904 & 0.9362 & 0.9444 & 0.9515 \\
\hline & & 0.8665 & 0.7487 & 0.9945 & 0.9945 & & 0.9884 & 0.9815 & 0.9713 & 0.9351 \\
\hline & & 1.0000 & 0.9902 & 0.9865 & 0.9985 & & 0.9924 & 0.8909 & 0.9175 & 0.9680 \\
\hline & \multirow{3}{*}{$\mathcal{S}_{6}$} & 0.9058 & 0.8467 & 0.9931 & 0.9989 & 0.9738 & & 0.9122 & 0.9124 & 0.9347 \\
\hline & & 0.8167 & 0.7058 & 0.9964 & 1.0000 & 0.9956 & & 0.9811 & 0.9949 & 0.9272 \\
\hline & & 0.9949 & 0.9876 & 0.9898 & 0.9978 & 0.9520 & & 0.8433 & 0.8298 & 0.9422 \\
\hline & \multirow{3}{*}{$\mathcal{S}_{7}$} & 0.9713 & 0.8860 & 0.9856 & 0.9956 & 0.9916 & 0.9869 & & 0.9704 & 0.9696 \\
\hline & & 0.9436 & 0.7927 & 0.9945 & 0.9971 & 0.9978 & 0.9927 & & 0.9727 & 0.9559 \\
\hline & & 0.9989 & 0.9793 & 0.9767 & 0.9942 & 0.9855 & 0.9811 & & 0.9680 & 0.9834 \\
\hline & \multirow{3}{*}{$\mathcal{S}_{8}$} & 0.9158 & 0.8302 & 0.9851 & 0.9949 & 0.9575 & 0.9927 & 0.9135 & & 0.9414 \\
\hline & & 0.8327 & 0.7109 & 0.9931 & 0.9971 & 0.9895 & 0.9927 & 0.9655 & & 0.9259 \\
\hline & & 0.9989 & 0.9495 & 0.9771 & 0.9927 & 0.9255 & 0.9927 & 0.8615 & & 0.9568 \\
\hline
\end{tabular}

TABLE I

EVALUATION RESULTS OF THE 8-FOLD CROSS-VALIDATION. THE RESULT OF ONE TRAINING-/TEST DATA COMBINATION INCLUDES IN THE FIRST ROW THE ACCURACY, IN THE SECOND ROW THE TRUE POSITIVE RATE AND IN THE THIRD ROW THE TRUE NEGATIVE RATE. THE MOST RIGHT COLUMN CONTAINS THE EVALUATION RESULTS FOR ONE TRAINING SET. THE OVERALL ACCURACY, TRUE POSITIVE AND TRUE NEGATIVE RATE WAS DETERMINED BY AVERAGING ALL RESULTS, THESE ARE $94.31 \%, 92.99 \%$ AND $95.63 \%$.

[7] R. Gallen, A. Cord, N. Hautière and D. Aubert, "Towards Night Fog Detection through use of In-Vehicle Multipurpose Cameras", Intelligent Vehicles Symposium, 2011, pp. 399-404.

[8] M.M. Gorkani and R.W. Picard, "Texture orientation for sorting photos at a glance", In Proceedings of the International Conference on Pattern Recognition, vol. 1, 1994, pp 459-464.

[9] A. Guerin-Dugue and A. Oliva, "Classification of scene photographs from local orientations features", Pattern Recognition Letters, vol. 21, 2000, pp. $1135-1140$.

[10] F.J. Harris, "On the use of windows for Harmonic Analysis with the Discrete Wavelet Transform", Proceedings of IEEE, vol. 66(1), 1978, pp. 51-83.

[11] N. Hautière, J.P. Tarel, J. Lavenant and D. Aubert, "Automatic fog detection and estimation of visibility distance through use of an onboard camera", Machine Vision and Applications, vol. 17, 2006, pp. 8-20.

[12] N. Hautière, R. Labayrade and D. Aubert, "Real-Time Disparity Contrast Combination for Onboard Estimation of the Visibility Distance", IEEE Transactions on Intelligent Transportation Systems, vol. 7(2), 2006, pp. 201-212.

[13] N. Hautière, R. Labayrade, C. Boussard, J.P. Tarel and D. Aubert, "Perception through Scattering Media for Autonomous Vehicles", Autonomous Robots Research Advances, 2008, pp.223-267.

[14] D. Marr, "Vision. A Computational Investigation into the Human Representation and Processing of Visual Information", W.H. Freeman and Company, 1982.

[15] W. Middleton, "Vision Through the Atmosphere", University of Toronto Press, 1952.

[16] A. Oliva, A. Torralba, A. Guerin-Dugue and J. Herault, "Global semantic classification using power spectrum templates", In Proceedings of The Challenge of Image Retrieval, 1999.

[17] A. Oliva and A. Torralba, "Modeling the Shape of the Scene: a Holistic Representation of the Spatial Envelope", International Journal of Computer Vision, vol. 42, 2001, pp. 145-175.
[18] D.A. Pomerleau, "Visibility Estimation from a Moving Vehicle Using the RALPH Vision System", IEEE Conference on Intelligent Transportation System, 1997, pp. 906-911.

[19] M.C. Potter, ”Meaning in visual search", Science, vol. 187, 1975, pp. 965-966.

[20] M.C. Potter, "Short-Term Conceptual Memory for Pictures", Journal of Experimental Psychology: Humans Learning and Memory, vol. 2(5), 1976, pp. 509-522.

[21] M.C. Potter, A. Staub and D.H. O'Conner, "Pictorial and conceptual representation of glimpsed pictures", Journal of Experimental Psychology: Human Perception and Performance, vol. 30, 2004, pp. 478-489.

[22] M. Szummer and R.W. Picard, "Indoor-outdoor image classification", IEEE International Workshop on Content-Based Access of Image and Video Databases, 1998, pp.42-51.

[23] A. Torralba and A. Oliva, "Semantic Organization of Scenes using Discriminant Structural Templates", Proceedings of IEEE International Conference on Computer Vision, vol. 2), 2001, pp. 1253-1258.

[24] A. Vailaya, A. Jain and H.J. Zhang. "On Image Classification: City Images vs. Landscapes", Pattern Recognition, vol. 31, 1998, pp.19211935.

[25] A. Vailaya, A. Jain, M. Figueiredo and H.J. Zhang. "Content-Based Hierarchical Classification of Vacation Images", In Proceedings of the IEEE International Conference on Multimedia Computing and Systems, vol. 2, 1999, pp. 518-523.

[26] World Meteorological Organization. "International Meteorological Vocabulary". WMO-No. 182, 1992. 